\title{
Near-infrared indocyanine green video angiography in aneurysm surgery
}

\author{
Aaron Mohanty \\ Division of Neurosurgery, Department of Surgery, University of Texas Medical Branch at Galveston Galveston, Texas, USA
}

\author{
Address for correspondence: \\ Dr. Aaron Mohanty, \\ 301 University Boulevard, \\ John Sealy Annex, 9.208, \\ Galveston, TX 77555-0517, USA. \\ E-mail: aarmohanty@yahoo.com
}

DOI: $10.4103 / 0028-3886.55568$

The most important goal of a vascular neurosurgeon during an intracranial aneurysm clipping is achieving complete exclusion of the aneurysm from the circulation with preservation of blood flow distal to the aneurysm. Occlusion of the parent vessel or distal branches due to improper clip application has been a significant factor in the poor outcome. Similarly, incomplete clipping of an aneurysm has been associated with aneurysmal growth and potential for future rupture. In the literature, partial aneurysm clipping with residual neck has been cited in $4-19 \%$ of cases while in an equally significant population (0.3-12\%) inadvertent occlusion of the major vessels occurs. Though in the latter group, surgery has been often performed to reposition the clip, there has been questionable improvement in the overall outcome.

Several techniques have been advocated to address the above concerns. The two most commonly used options are intraoperative angiography and intraoperative microvascular Doppler evaluation of the distal vessels. In the recent years, intraoperative near-infrared indocyanine green (ICG) video angiography has been considered in addition to the above two options. Initially described by Rabbe et al., in 2003, this has been emerging as an important intraoperative adjunct in assessing the flow distal to the aneurysm. ${ }^{[1]}$ In recent years, technological advances have facilitated the integration of video angiography into the existing microscope systems, thus considerably simplifying the procedure and reducing the overall operative time.

Indocyanine green, a fluorescent dye was initially made available in the mid-1970s for ophthalmological usage.
After an intravenous injection, within few seconds the dye binds to the globulins and stays entirely in the intravascular compartment. It has a half life of 3-4 min in plasma and is subsequently excreted by the liver. It has an absorption peak at $805 \mathrm{~nm}$ and an emission peak at $835 \mathrm{~nm}$. During the procedure, the dye is injected intravenously in a dose of $0.2-0.5 \mathrm{mg} / \mathrm{kg}$ of body weight and the field of interest is illuminated with near-infrared light which induces the fluorescence of the dye. The emitted signal is captured, evaluated and can be recorded.

In an article presented in this issue, the authors report their experience with 208 ICG video angiographic studies in 120 patients. ${ }^{[2]}$ Diagnosis of abnormal findings was evident in 10 patients, of which four had residual aneurysms, and six had inadvertent occlusion of parent or branching artery. This led the authors to reapply the clips in all except in one; in whom though the images suggested the abnormality, it was misinterpreted as normal, ultimately resulting in a fatal outcome. On comparison of the ICG video angiography and postoperative digital subtraction angiography (DSA) findings, the authors found a $92.6 \%$ concordance rate between the intraoperative ICG and the postoperative DSA. Of the inconsistencies, three had mild postoperative stenosis in DSA while in another three there was incomplete clipping of the aneurysm.

The study corroborates similar results published in recent literature. Rabbe et al., ${ }^{[3]}$ reported good correlation of the results of ICG video angiography with intra 
or postoperative DSA in $90 \%$ of cases. Of the $10 \%$ of patients where it did not correspond, only in $2.7 \%$ of cases the missed findings either were significant stenosis or residual aneurysm necks. More importantly, in 9\% cases, the findings observed at ICG video angiography led the authors to consider clip reapplication. Similar results have also been reported by others. ${ }^{[4]}$

In another study, de Oliveira et al. further extended the utility of the study to assess the flow in perforating arteries by video ICG during intracranial aneurysm surgery. They analyzed the involvement of perforating arteries during the surgical dissection and clip application. The authors concentrated only on the patency of the small arteries that were not visible in the preoperative DSA. The intraoperative patency of the vessels was compared with the development of infarctions in the postoperative computed tomographic scans. The authors reported a high sensitivity of the technique which led to appropriate treatment alterations during the surgical procedure improving the overall results.

However, there have been occasions where the ICG angiography has been reported to have provided a false sense of security. Anatomy outside the microscope's viewing area is not detected during the evaluation. Additionally, blood in the operative field and arachnoid scarring add to misinterpretation of the results thus further restricting its usefulness. Additionally, deep-seated aneurysms such as those located in the anterior communicating aneurysm complex have been difficult to assess as they have been reported to be associated with higher neck residuals than surface aneurysms. ${ }^{[4]}$ Calcifications and thick-walled atherosclerotic vessels or partially or completely thrombosed aneurysms also impair adequate visualization thus limiting its usefulness. Additionally, as illustrated by the authors in the present article, misinterpretation of image findings is a likely possibility, especially with untrained eyes.

Despite these limitations, the most important utility of ICG video angiography has been in providing immediate real-time information to assess the blood flow in vessels. It also assesses if the aneurysm has been partially or satisfactorily obliterated. The difficulties in interpreting the results with complex, giant and deep-located aneurysm is a distinct disadvantage, especially considering that in these cases, the surgeon needs additional guidance in proper clip application.

Does this indicate that ICG video angiography would ultimately replace intraoperative Doppler evaluation of the distal vessels or intraoperative DSA? Though considered to be the "gold standard", intraoperative DSA is time-consuming, expensive and requires additional an professional in the operative suite. Intraoperative microvascular Doppler ultrasonography though can detect flow in distal vessels is affected by coexisting vasospasm and does not detect inclusion of small perforators in the clip. Additionally, inability to detect remnants of aneurysm neck is a further limitation. Considering these, ICG video angiography appears promising in detecting the distal flow and assessing the completeness of the aneurysm clipping. Despite its above mentioned limitations, it advantages appear to outweigh the inefficacies. Though not sure at this point, it is to be seen if ICG video angiography can replace the time-consuming intraoperative angiography. Till then, it certainly complements the existing armamentarium and gives the surgeon an additional option.

\section{References}

1. Rabbe A, Beck J, Gerlach R, Zimmermann M, Seifert V. Near infrared indocyanine green video angiography: A new method for intraoperative assessment of vascular flow. Neurosurgery 2003;52:132-9.

2. Li J, Lan Z, He M, You C. Assessment of microscope-integrated indocyanine green angiography during intracranial aneurysm surgery: A retrospective study of 120 patients. Neurol India 2009;453-9.

3. Rabbe A, Nakaji P, Beck J, Kim LJ, Hsu FP, Kamerman JD, et al. Prospective evaluation the surgical microscope integrated intraoperative near infrared indocyanine green video angiography during aneurysm surgery. J Neurosurg 2005;103:982-9.

4. Dashti R, Laakso A, Niemela M, Porras M, Hernesniemi J. Microscopeintegrated near infrared indocyanine green video angiography during surgery of intracranial aneurysms: The Helsinki experience. Surg Neurol $2009 ; 71: 543-50$.

Accepted on 06-08-2009

Source of Support: Nil, Conflict of Interest: None declared. 